VIERA, M.C. Aulacomnium palustre var. imbricatum B.S.G. Novedad para la brioflora española Bol. Soc. Brot. ser. 2, 60:125-128 (en prensa).

\title{
ALGUNAS ESPECIES DE BRIOFITOS NUEVAS DEL SURESTE DE ESPAÑA
}

\author{
Rosa Ma. ROS \& Juan GUERRA
}

RESUMEN: Se mencionan 21 especies de briófitos, algunos de ellos desconocidos en el sur o sureste español y otros que, aunque han sido citados anteriormente en este área, son raros o infrecuentes. Los más interesantes son los que se mencionan en el resumen en inglés. También se aportan datos concernientes a la corologia de algunas especies.

SUMMARY: Twenty one species of bryophytes, some of them new for the flora of $S$ or SE Spain and others uncommon are reported. The most interesting records are: Acaulon dertosense, Anomodon viticulosus, Bryum klinggraeffii, Entosthodon attenuatus, Leptobarbula berica, Phascum floerkeanum and Pterygoneurum lamellatum. Data are also presented referring to the corology of some species.

Son varios los articulos publicados hasta el momento por nosotros dentro de esta línea de trabajo, orientados a dar a conocer las novedades briofloristicas del sureste español: Alcaraz \& a1. (1982), Ros \& Llimona (1984) y Ros \& Guerra (1985). En éste presentamos los hallazgos más recientes, especies terricolas en su mayor parte, pertenecientes a 1a provincia de Murcia. Hemos recogido, en los casos más interesantes, los datos corológicos conocidos hasta el momento y realizado un mapa de distribución peninsular. La nomenclatura empleada ha sido la de Casas (1981) y Grolle (1983), para musgos y hepáticas respectivamente. Todas las muestras se hayan depositadas en el herbario del Departamento de Biología Vegetal (Botánica) de la Universidad de Murcia (MUB). 
Acaulon dertosense Casas, Sérgio, Cros \& Brugués

En suelos básicos, a veces nitrificantes y enriquecidos en sales. En condiciones, pues, similares a las descritas por Casas \& al. (1986). Aparece mezclado con otros pequeños briófitos como Pterygoneurum ovatum, Aloina ambigua, Anisothecium howei y Pottia sp. pl.

MURCIA: El Carrascalejo (Bullas), XH1313. Rambla de la Herreña (Mula), XH2204. Cruce Cartagena-Los Narejos (San Javier), XG9172. Cabezo de la Fuente, Los Belones (Cartagena), XG9665. C: Cabezo Coroneles, Sierra de la Torrecilla (Lorca), XG0474. Barranco Blanco, carretera Caravaca-Lorca (Caravaca), WH 9402. Estación de Ulea (Ulea), XH 5555, Carretera Murcia-San Javier, Km 6, XG 7091.

Sólo se conocía, hasta el momento, de la provincia de Tarragona en el Puerto de Besseit: Xerta (Casas, Cros, Brugués \& Sérgio, 1985) y Pauls dels Ports (Casas \& al., 1986). Mapa 1.

Anomodon viticulosus (Hedw.) Hook. \& Tayl.

En roca caliza vertical y sombreada. Con Leptodon smithii y

Schistidium apocarpum. Nueva cita para el sureste peninsular. WH8829.

MURCIA: Cenajo del Agua Cernida, Sierra de los Alamos (Moratalla),

Según Puche (1986), la localidad más meridional conocida hasta ahora era la de Valencia.

Bryum flaccidum Brid.

Generalmente coloniza protosuelos en hendiduras de rocas calizas con alto contenido en materia orgánica. Acompañada por Trichostomum brachydontium, Didymodon sp. pl. y otras potiáceas. En el sureste español ha sido citada únicamente en la provincia de Albacete (Jiménez, Ros \& Guerra, 1986) y aunque se tienen datos de algunas localidades españolas, todos ellos son de la mitad oriental de la Península Ibérica.

MURCIA: Sierra del Carche (Jumilla), en el Pico El Carche, XH6055 y Barranco del Pozo de la Nieve, XH5855. Puerta de Moratalla, Río Benamor (Moratalla), WH9130. Rambla de Enmedio, Sierra de Burete (Cehegín), XH1013. Venta del Pino (Cehegín), XH1006. Sierra de Espuña, Río Espuña (Totana) XH2790. Carretera Los Alcázares-San Javier (San Javier), XG9084. Fuente de la Muela (Cartagena), XG6861. Alto del Peral, Sierra de la Almenara (Lorca), XG2758. Sierra de la Torrecilla (Lorca): Cabezo Colorado, XG0473 y c. Cabezo Coroneles, XG0474.

Bryum gemmilucens Wilck. \& Demar.

En suelos carbonatados y pobres en materia orgánica, generalmente nitrificados, en ramblas y bordes de carreteras. Se presenta a menudo con Bryum argenteum y Anisothecium howei. Es novedad para el sureste de España. MURCIA: Puerta de Moratalla, WH9130. Las Oicas, Archivel (Caravaca), WH8915. El Carrascalejo (Bullas), XH1313. El Aceniche (Bullas), XH1305. Rambla de la Herreña (Mula), XH2204. El Carmolí (Cartagena), XG9073. Sierra de la Almenara, c. Campico-López (Lorca), XG2762.

ALBACETE: Sierra del Relumbrar (Alcaraz), Cerro de la Calva, WH 2673; c. Finca el Palmar, WH 3178; Finca el Palomar, WH 3278; Arroyo Barbezoso (Villapalacios), WH 2471; vertiente SO, WH 2070; Casa de la Cueva, WH 2771. La distribución ibérica de esta especie es poco conocida dado lo reciente de su descripción (Wilczek \& Demaret, 1976). Unicamente se conocen las citas siguientes. Sérgio \& al. (1984) en Portugal (Algarve). Casas, Brugués \& Peñuelas (1983) en Gerona: Cap Norfeu. Casas y Brugués (1983a) en Tarragona: entre Vilarba dels Arcs y la Pobla de Massaluca y en Cáceres: Jaraicejo. 
Bryum klinggraeffii Schimp.

Se ha encontrado en taludes ácidos sobre areniscas y cuarcitas, generalmente protegidos por arbustos y en una pared caliza en la que se ha acumulado tierra. Nueva cita para la provincia de Murcia.

MURCIA: Fuentes del Río Mula (Bullas), XH1409. Cabezo de la Jara (Puerto Lumbreras), WG9576.

Las localidades españolas conocidas hasta ahora son las siguientes. Casas, Fuertes, Simó \& Varo (1982) en Teruel: Valdelinares (Sierra de Gúdar). Jiménez, Ros \& Guerra (1986) en Albacete: Campamento de San Juan (Sierra del Calar del Mundo). Mateo \& Varo (1987) en Granada: Sierra de Baza. Miguel \& Ederra (1987) en Navarra: Urdiain. Mapa 2.

Entosthodon attenuatus (Dicks.) Bryhn.

En suelo de ladera cercana al mar y protegido por plantas herbáceas. Mezclada con otros musgos como Tortula vaaliana, Barbula unguiculata y Didymodon vinealis. Novedad para el sureste español.

MURCIA: Cabezo de la Fuente, Los Belones (Cartagena), XG9665.

Entosthodon curvisetus (Schwaegr.) C. Milll.

Encontrada en protosuelos formados en la base de rocas y hendiduras, acompañada de Fissidens incurvus y Targionia hypophylla entre otras. Es una especie relativamente rara en la Península, desconocida en la mitad septentrional y escasamente citada. Existen, sin embargo, numerosas localidades en Baleares. Aportamos una nueva en el sureste peninsular que representa la segunda para la provincia de Murcia, donde había sido citada por Allorge \& Allorge (1946).

MURCIA: Cabezo de la Fuente, Los Belones (Cartagena), XG9665.

Entosthodon pallescens Jur.

Concavidades en la base de rocas calizas situadas en el fondo de un barranco, formando un extenso césped junto a Fossombronia caespitiformis, Cephaloziella baumgartneri y Funaria pulchella entre otras especies. Nueva cita para el sureste español.

MURCIA: Sierra de la Muela (Cartagena), XH6862.

En España presenta una distribución de tipo mediterráneo. Las localidades en las que ha sido citada (englobada la var. mitratus (Cas. Gil) Wijk \& Marg.) son las que se mencionan a continuación. Geheeb (1874) en Cádiz: Jerez. Warnstorf (1911) en Sevilla. Armitage (1928) en Málaga: Villanueva de la Concepción. Montserrat (1953) en Menorca: Algendar. Cortés-Latorre (1958) en Zaragoza: Monasterio de Piedra y recopila varias citas anteriores, incluídas las que fueron publicadas bajo el sinónimo de la var. mitratus, (=Entosthodon physcomitrioides). Casares \& Beltrán (1912) en Madrid: Vaciamadrid. Koppe (1965) en Mallorca: Sóller. Casas (1979) en Menorca: Mahón. Casas \& Brugués (1983b) en Menorca: Port de Ciutadella. Mapa 3.

Fissidens exilis Hedw.

Especie bastante rara en el sureste, pues solo ha sido encontrada en una ocasión en el suelo de una cueva, acompañada de Trichostomum crispulum. Es novedad para la flora del sureste de la Península.

MURCIA: Sierra de la Pila, Barranco de las Cabras (Abarán), XH5337.

Las localidades españolas que se conocen son las siguientes. Colmeiro (1849) en Madrid: Casa de Campo y Jardín Botánico. Beltrán-Bigorra (1929) en Castellón: Nules. Allorge \& Casas (1968) en Gerona: c. Olot. Brugués (1978) en Tarragona: Estret de la Vall (Montblanc). Vives (1978) en Valencia: Plana Baixa. Cros (1982) en Formentera: Pla del Rey. Cros \& Rosselló (1984) en Formentera. Mapa 4.

Fissidens incurvus Starke ex Röhl

En la base de rocas y fisuras con varias especies de Weissia y 
Trichostomum. Encontrada a menudo fértil y por ello fácilmente determinable. Nueva cita para el sureste español.

MURCIA: El Carrascalejo (Bullas), XH1313. Cabezo de la Fuente, Los Belones (Cartagena), XG9665. Sierra de la Muela (Cartagena), XG6862 y XG6861. Cabezo de la Jara (Puerto Lumbreras), WG9756.

Grimmia laevigata (Brid.) Brid. Sobre rocas metamórficas en lugares expuestos y desprotegidos, formando pequeñas almohadillas aisladas. Novedad para la provincia de Murcia.

MURCIA: Sierra de la Torrecilla, Rambla del Hortillo (Lorca), XG0372, a $600 \mathrm{~m}$.

Grimmia tergestina Tomm. ex B.S.G.

En rocas calizas soleadas, a $1100 \mathrm{~m}$ de altitud. Se trata de una nueva cita para el sureste español.

MURCIA: Valle del Río Espuña, Sierra de Espuña (Totana), XG2890.

La localidad más próxima conocida es la de la provincia de Alicante:

Planes de la Baronia, cerca del desvio a Catamarruc (Casas \& al., 1984).

Leptobarbula berica (De Not.) Schimp.

En una fisura de roca caliza con muy poco espesor de suelo. Junto a Gymnostomum luisieri y pequeñas hepáticas como Cephaloziella baumgartneri y

Southbya nigrella. Ampliamos su distribución hacia el sur de España. MURCIA: Sierra de la Muela (Cartagena), XG6862.

El mapa de distribución peninsular ha sido realizado recientemente por Casas, Brugués, Cros \& Sérgio (1985), al cual se ha de añadir esta nueva cita. Mapa 5 .

Leptodictyum kochii (B.S.G.) Warnst.

En un canal de riego. Ocasionalmente sumergida. Novedad para el sureste español.

MURCIA: Huerta de Murcia, XH6407.

Orthotrichum rupestre Schwaegr.

En rocas de carácter ácido, ligeramente protegidas por arbustos, a

$1000 \mathrm{~m}$ de altitud. Es la primera cita en el sureste de España.

MURCIA: Sierra de Carrascoy, Rambla de Roy (Totana), XG51190.

Phascum floerkeanum Web. \& Mohr

En suelos denudados, secos y margosos, a veces salinos o yesíferos (conductividad de 3,2-3,4 mmhos/cm y pH de 7,6-7,8). Generalmente aparecen ejemplares aislados entre pequeños musgos como Acaulon triquetrum y Pottia sp. pl., etc. Debido a su pequeño tamaño y a su carácter anual es muy fácil que pasen desapercibidos. Estas citas son las primeras en el sur de España. MURCIA: Barranco Blanco, carretera Caravaca-Lorca (Caravaca), WH9402. El Aceniche (Bullas), XH1305. Cruce Cartagena-Los Narejos (San Javier), XG8981. Sierra de la Torrecilla, c. Cabezo Coroneles (Lorca), XG0474.

Las citas anteriores de esta especie en España son las siguientes, Luisier (1915) en Salamanca: en la carretera de Zamora, c. de Aldeaseca. Casas, Seró, Ubach \& Vives (1956) en Barcelona: Bruguérs y Prat de Llobregat. Casas (1970) en Zaragoza: Bujaraloz, Hostal del Ciervo. Fuertes \& García-Gómez (1985) en Navarra: Caparroso. También conocemos una localidad inédita de la provincia de Málaga: c. Mijas. Mapa 6.

Pterygoneurum lamellatum (Lindb.) Jur.

Coloniza los suelos arcillosos o margosos, a veces enriquecidos en sales y por tanto de alta conductividad (1-4 mmhos/cm) y pH de 7,4-8. Aparece con Crossidium crassinerve, Bryum argenteum, Bryum bicolor y Didymodon sp. pl. 


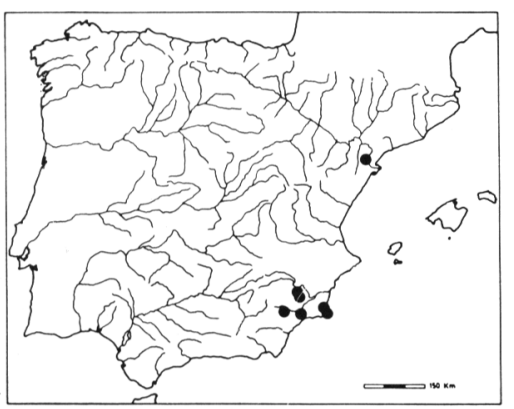

Mapa 1

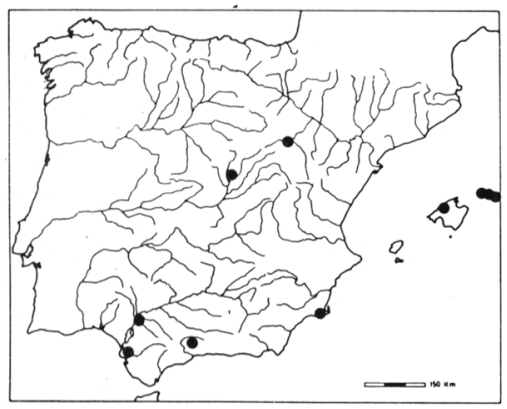

Mapa 3

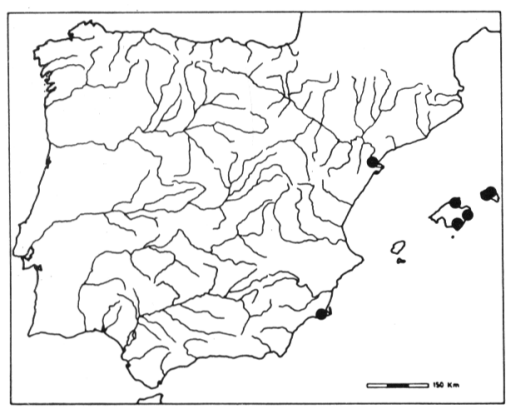

Mapa 5

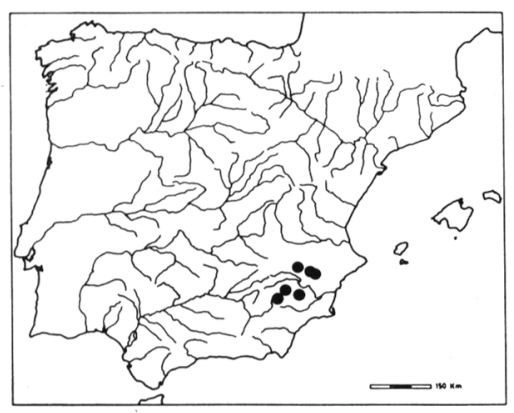

Mapa 7

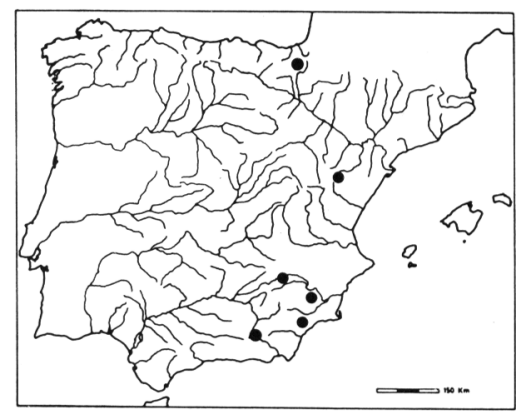

Mapa 2

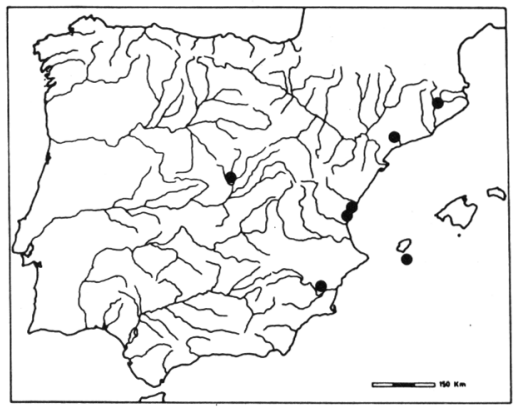

Mapa 4

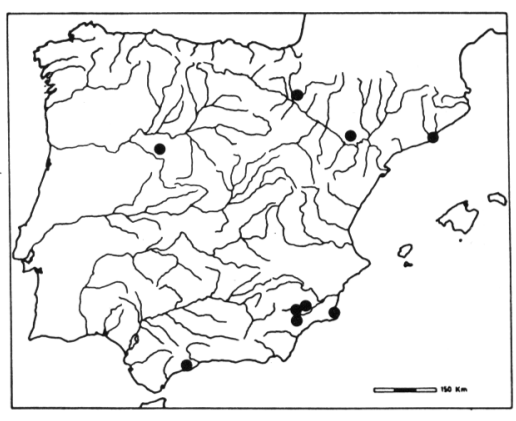

Mapa 6

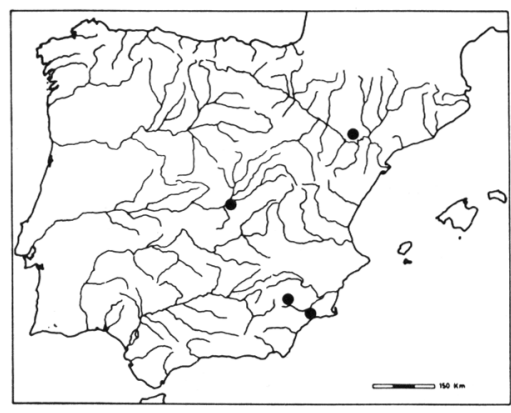

Mapa 8 
Es una especie rara en la Península Ibérica, sin embargo, en áreas de tendencia continental del sureste español es relativamente frecuente. Nueva cita para Murcia.

MURCIA: Cabezo de la Rosa (Jumilla), XH5555. Cerro de la Sal (Jumilla), XH4360. Loma Ancha, Archivel (Caravaca), WH8616. Cerro de Mantilla, en la carretera a Moralejo (Caravaca), WH7605. Salto Lucero (Bullas), XH1709. Molino de Sebastián (Bullas), XH1810.

La única cita que se conocía hasta ahora en España es la de Rungby (1964) en Albacete: Hellín y Tobarra. Pese a la proximidad de nuestras localidades con ésta, pensamos que son de interés, pues así se confirma su presencia en la Península Ibérica. Mapa 7.

Pterygoneurum sampaianum (Mach.) Mach.

Encontrada en suelos secos, margoso-yesíferos, con conductividad medianamente alta $(3,4-3,6 \mathrm{mmhos} / \mathrm{cm})$ y salinos en la que ésta es algo mayor ( 5 mmhos $/ \mathrm{cm}$ ) y pH entre 7,5 y 7,6. Junto a Pottia sp. pl., Crossidium crassinerve, Bryum bicolor y en alguna ocasión con Pterygoneurum ovatum. En esta última observación diferimos de lo manifestado por Casas \& Brugués (1978), referente $a^{\prime}$ que estas dos especies de Pterygoneurum no se presentan en la misma época. La coincidencia fenológica, al menos en el sureste, parece muy clara. Las localidades que se mencionan son las primeras en el cono suroriental de la Península Ibérica.

MURCIA: Barranco Blanco, en la carretera Caravaca-Lorca (Caravaca), WH9402. Carretera de Mazarrón a Aguilas (Mazarrón), XG4561.

Las citas que se conocen de esta especie, hasta el momento, son las que siguen. Machado (1925) en Portugal: Algarve, c. Portimâo. Casas \& Brugués (1978) en Zaragoza: Los Monegros. Casas (1966 Ed.) en Toledo: Añover de Tajo (Leg. E. Fuertes). Mapa 8.

Riccia crozalsii Levier

Se ha encontrado en suelos secos de conductividad ligeramente alta (3,9 mmhos/cm) y pH de 7,5, protegidos por pequeñas herbáceas. Las especies acompañantes eran Aloina bifrons, Pseudocrossidium hornschuchianum, Didymodon aaronis, etc. Se triata de una novedad para el sureste español.

MURCIA: Castillo de Mula, XH3212. Carretera de Murcia a San Javier, Km 6 (Murcia), XG7091.

Riccia michelii Raddi

En suelo arcilloso-arenoso y ligeramente protegido por arbustos. Junto a Riccia sorocarpa, Bryum dunense y Pseudocrossidium hornschuchianum, entre otras especies. Cita nueva para el sureste de España.

MURCIA: Calblanque, Salinas del Rasall (Cartagena), XG9964.

Weissia longifolia Mitt.

En suelos descubiertos, formando densos céspedes junto a Didymodon trifarius y Aloina sp. Ya fué citada por Casas (1973) en la provincia de Murcia, pero desde entonces no se había vuelto a recolectar, se trata, por tanto, de un hallazgo interesante por la poca frecuencia con que se encuentra.

MURCIA: Peña Rubia (Cehegín), XH0416.

Su distribución en España es claramente oriental, pues solo ha sido detectada en zonas más o menos cercanas a la costa mediterránea.

\section{AGRADECIMIENTOS}

Agradecemos a C. Casas y M. Brugués la revisión de los ejemplares pertenecientes al género Acaulon y a Entosthodon pallescens, así como la confirmación de las muestras del génerọ Pterygoneurum. A C. Sérgio, F. 
Demaret y J.P. Hébrard por la revisión de los taxones Leptobarbula berica, Bryum gemmilucens y Leptodictyum kochii respectivamente.

\section{BIBLIOGRAF IA}

ALCARAZ, F., ROS, R.M., EGEA, J.M. \& LLIMONA, X. -1982- Contribución al conocimiento de la flora briofitica del sureste de España. Collect. Bot., 13 (1):129-142.

ALLORGE, V. \& ALLORGE, P. -1946- Notes sur la flore bryologique de la Péninsule Ibérique $X$. Muscinées du Sud et de l'Est de l'Espagne. Rev. Bryol. Lichénol., 15:175-200.

ALLORGE, V. \& CASAS, C. -1968- Contribución al estudio de la flora briológica catalana. II. Briófitos del llano de olot y montañas próximas. Collect. Bot., 7:47-68.

ARMITAGE, E. -1928- Some Spanish Bryophytes. The Bryologist, 31:123-126.

BELTRAN-BIGORRA, F. -1929- Adiciones a las muscíneas de la provincia de Castellón. Mem. R. Soc. Esp. Hist. Nat., 15:275-284.

BRUGUES, M. -1978- Flora briológica de los estratos del Buntsandstein de la Cordillera Costero Catalana. Rev. Bryol. Lichénol., 44 (2):149-201.

CASARES-GIL, A. \& BELTRAN-BIGORRA, F. -1912- Entosthodon physcomitrioides nov. sp., Bol. R. Soc. Esp. Hist. Nat., 12:375-377.

CASAS, C. -1970- Avance sobre el estudio de la flora briológica de Los Monegros (Valle Medio del Ebro). Notulae Bryologicae I. Acta Phytotax. Barc., 6:5-12.

CASAS, C. -1973- Datos para la flora briológica española. Algunos musgos y hepáticas del sureste de España. Rev. Fac. Ciênc. Lisboa. 17 (2) : 603-616.

CASAS, C. -1979- Funaria pallescens (Jur.) Broth. var. mitratus (Cas.-Gil) Wijk et Marg. en Menorca. Rev. Bryol. Lichénol., 45 (4):467-470.

CASAS, C. -1981- The mosses of Spain, an annotated check-list. Treb. Inst. Bot. Barcelona, 7:1-57.

CASAS, C. (Ed.) -1986- Brioteca Hispánica. Acta Bot. Malacitana, 11:83-112.

CASAS, C. \& BRUGUES, M. -1978- Nova aportació al coneixement de la brioflora dels Monegros. Anales Inst. Bot. Cavanilles, 35:102-114.

CASAS, C. \& BRUGUES, M. -1983a-Addicions a la brioflora de les comarques tarragonines. Collect. Bot., 14:235-241.

CASAS, C. \& BRUGUES, M. -1983b- Contribució a la brioflora de l'illa de Menorca. Collect. Bot., 14:231-234.

CASAS, C., BRUGUES, M., CROS, R.M. \& SERGIO, C. -1985- Cartografia de Briòfits. Península Ibèrica i les illes Balears. Canàries. Açores i Madeira. Institut d'Estudis Catalans. Barcelona.

CASAS, C., BRUGUES, M. \& PEÑUELAS, J. -1983- Briòfits de l'Alt Empordá. Annals. Inst. Est. Empordanesos, 16:15-32.

CASAS, C., CROS, R.M., BRUGUES, M., SERGIO, C. \& SIM-SIM, M. -1984- Estudio de la flora briofitica de las comarcas alicantinas. Anales de Biología, 2 (Sección especial, 2):215-228.

CASAS, C., CROS, R.M., SERGIO, C. \& SIM-SIM, M. -1985- Estudio de la brioflora dels Ports de Beseit. Orsis, 1:13-31.

CASAS, C., FUERTES, E., SIMO, R.M. \& VARO, J. -1982- Aportación al conocimiento de la flora briológica española. Nótula IV: Las Sierras de Jabalambre y Gúdar (Teruel). Acta Bot. Malacitana, 7:119-140.

CASAS, C., SERGIO, C., CROS, R.M. \& BRUGUES, M. -1986- Acaulon dertosense sp. nov., musgo terrícola de los olivares del Baix Ebre (Cataluña). Anales Jard. Bot. Madrid, 42 (2):229-301.

CASAS, C., SERO, P., UBACH, M. \& VIVES, J. -1956-Flora briológica de las comarcas barcelonesas. Collect. Bot., 5:119-141.

COLMEIRO, M. -1849- Apuntes para la flora de las dos Castillas. Madrid. 
CORTES-LATORRE, C. -1958- Aportaciones a la Brioflora española. Una rápida visita al Monasterio de Piedra. Anal. Inst. Bot. Cavanilles, 15:491-198.

CROS, R.M. -1982- Algunos briófitos interesantes para la flora Balear. Acta Bot. Malacitana, 7:141-150.

CROS, R.M. \& ROSSELLO, J.A. -1984- An approach to the bryophytic flora of the Pityusic Islands. In: Kubier, H., Alcover, J.A. \& Guerau d'Arellano Tur (Eds.). Biogeography and Ecology of the Pityusic Islands.

FUERTES, E. \& GARCIA-GOMEZ, -1985- Flora muscinal gipsícola y sus comunidades, dependientes de la vegetación vascular, en Navarra (España). Cryptogamie, Bryol. Lichénol., 6 (2):95-120.

GEHEEB, A. -1984- Beitrag zur Moosflora von Spanien. Flora, 33:1-6.

GROLLE, R. -1983- Hepatics of Europe including the Azores: an annotated list of species, with synonyms from the recent literature. J. Bryol., $12: 403-459$.

JIMENEZ, M.N., ROS, R.M. \& GUERRA, J. -1986- Flora y vegetación briofítica des sector noroccidental de la Sierra del Calar del Mundo (SW de Albacete, España). Acta Bot. Malacitana, 11:113-146.

KOPPE, F. -1965- Bryologische Beobachtungen auf der Insel Mallorca. Botaniska Notiser, 118:25-48.

LUISIER, A. -1915- Fragments de Bryologie Ibérique, 6. Sur la distribution géographique de Triquetrella arapilensis Luis. 8. Espèces nouvelles pour la flore espagnole. Broteria Ser. Bot., 13:150-151; 154-157.

MACHADO, A. -1925- Sinopse das Briofitas de Portugal. Lisboa.

MATEO, F.D. \& VARO, J. -1987- La vegetación briofítica de la Sierra de Baza (Granada). Act. VI Simp. Nac. Bot. Cript.:537-846.

MIGUEL, DE A. \& EDERRA, A. -1987- Estructuras reproductivas asexuales de diversas especies de musgos de Navarra (España). Act. VI Simp. Nac. Bot. Cript.:557-565.

MONTSERRAT, P. -1953- Algunas briófitas de Menorca. Anal. Inst. Bot. Cavanilles, 12:395-399.

PUCHE, F. -1986- Sobre la presencia de algunas especies de briófitos nuevos para el levante español. Cryptogamie, Bryol. Lichénol., 7 (3):235-248.

ROS, R.M. \& GUERRA, J. -1985- Aportación al conocimiento de la brioflora del sureste de España. Anales Jard. Bot. Madrid, 41: (2):257-266.

ROS, R.M. \& LLIMONA, X. -1984- Estudio briológico del sistema de sierras de Ponce y Quípar (Oeste de Murcia, Sureste de España). Collect. Bot., $15: 431-457$.

RUNGBY, S. -1964- A contribution to the Bryophytic Flora of Spain, Especially the environs of Hellín. Botaniska Notiser, 117:167-172.

SERGIO, C., SIM-SIM, M., CASAS, C., CROS, R.M. \& BRUGUES, M. -1984- A vegetaçao briológica das formaçoes calcarias de Portugal II. Bol. Soc. Brot., 57 (2 a ser.) :257-307.

VIVES-CODINA, J. -1976- Aproximació a la flora dels briòfi.ts Balears. Barcelona, 64 pág.

VIVES-CODINA, J. -1978- Aproximació a la flora dels briòfits del Migjorn Valencià. Barcelona. 69 pág.

WARNSTORF, C. -1911- Verzeichnis der von M. Fleischer 1908 während der Monate April und Mai in Süfrankreich und Spanien beobachteten Laub-, Leber-und Torfmoose. Hedwigia, 50:189-203.

WILCZEK, R. \& DEMARET, F. -1976- Les espèces belges du "complexe Bryum bicolor" (Musci). Bull. Jard. Bot. Nat. Belg., 46:511-544.

(Aceptado para su publicación el 30 de marzo de 1987)

Dirección de los autores: Departamento de Biología Vegetal (Botánica). Facultad de Biología. Universidad de Murcia. 